\title{
A COMPONENT OF GENETIC VARIATION AMONG MICE IN ACTIVITY OF TRANSMEMBRANE METHYLTRANSFERASE I DETERMINED BY THE $H$-2 REGION
}

\author{
JASNA MARKOVAC* and RoBERT P. ERICKSON† \\ Department of Human Genetics, University of Michigan School of Medicine, Ann Arbor, MI, 48109- \\ 0010, U.S.A.
}

(Received 9 August 1984; accepted 11 February 1985)

\begin{abstract}
The effects of the mouse major histocompatibility complex, $H-2$, on phospholipid methyltransferase I and II activities were investigated on hepatocyte membranes from inbred, congenic and recombinant strains. Each methyltransferase was assayed individually by measuring the incorporation of radiolabel from $S$-adenosyl-L-[ methyl $\left.^{-3} \mathrm{H}\right]$ methionine into endogenous phospholipids. Our results indicate that $H-2$ exerted a significant effect on methyltransferase I but not on methyltransferase II activity. Thus, as in lower eukaryotes, two distinct enzymes were involved in methylation of phosphotidylethanolamine (PE) to phosphatidylcholine (PC). In addition, this effect was localized to the $\mathrm{K}$ end of the major histocompatibility complex by the use of recombinant haplotypes.
\end{abstract}

The mouse major histocompatibility complex, $\mathrm{H}-2$, has been shown to influence intracellular cyclic AMP levels and glucagon binding to hepatocyte membranes $[1,2]$. It has also been suggested that the sequential methylation of membrane phospholipids mediates the transduction of a variety of receptormodulated signals [3]. In view of these findings, we investigated the effects of $H \cdot 2$ on phospholipid $\mathrm{N}$ methylation in liver cell membranes from inbred strains of mice. The synthesis of phosphatidylcholine from phosphatidylethanolamine appears to involve two specific methyltransferases (MT I and MT II) which mediate the stepwise transfer of three methyl groups from $S$-adenosyl-L-methionine to the phospholipid intermediates [4]. By measuring the incorporation of radiolabel from $S$-adenosyl-L$\left[\right.$ methyl ${ }^{3} \mathrm{H}$ ]methionine into endogenous phospholipids, we were able to assay each methyltransferase individually in hepatocyte membranes from inbred and $H-2$ congenic mice. Our results indicate that $H$ 2 exerted a significant effect on mouse MT I activity but not on MT II, supporting the theory that, as in lower eukaryotic organisms such as Saccharomyces cerevisiae [5] and Neurospora crassa [6], two enzymes are involved in transmembrane methylation. Furthermore, the $\mathrm{H}$-2 effect was shown to map at the $\mathrm{K}$ end of the major histocompatibility complex.

\section{MATERIALS AND METHODS}

Unless otherwise indicated, the mice used were inbred from original stocks obtained from Jackson

* Present address: Department of Microbiology, Molecular Biology Institute, University of California, Los Angeles, CA 90024.

+ Address all correspondence to: Robert P. Erickson, M.D., Department of Human Genetics, Box 015, University of Michigan Medical School, 1137 East Catherine St. Ann Arbor, MI 48109-0010.
Laboratories, Bar Harbor, ME. A.TL and A.TH mice were gifts from Dr. John Neiderhuber, University of Michigan. All other recombinant haplotypes were donated by Dr. Jane Schultz, University of Michigan.

Livers were rapidly excised from animals killed by cervical dislocation and minced finely in ice-cold $1 \mathrm{mM} \mathrm{NaHCO}_{3}, \mathrm{pH} 7.4$. The suspension was homogenized in a Teflon-glass homogenizer for five strokes at low speed with a Thomas model 45 homogenizer and centrifuged for $1 \mathrm{~min}$ at $500 \mathrm{~g}$. The pellet was discarded, and the supernatant fraction was recentrifuged for $10 \mathrm{~min}$ at $1500 \mathrm{~g}$. The resulting pellet was quick-frozen in a dry ice-ethanol bath and stored at $-20^{\circ}$ until use.

The pellets were thawed by vigorous shaking in the homogenization buffer, $50 \mathrm{mM}$ 4-(2-hydroxyethyl)-1-piperazine-ethanesulfonic acid (HEPES), $10 \mathrm{mM} \mathrm{NaF}$, and $15 \mathrm{mM}$ ethyleneglycolbis(aminoethylether)tetra-acetate (EGTA), $\mathrm{pH} \quad 7.4$ [7]. Methyltransferase activities were determined essentially as described by Hirata et al. [4]. The methylation rate of endogenous phosphatidylethanolamine (PE) by MT I was obtained by measuring the incorporation of radiolabel from $S$-adenosyl-L-[methyl$\left.{ }^{3} \mathrm{H}\right]$ methionine $\left(\left[{ }^{3} \mathrm{H}\right] \mathrm{SAM}\right)$ into phosphatidylmonomethylethanolamine (PMME). The reaction medium contained $10 \mathrm{mM} \mathrm{MgCl} 2,0.1 \mathrm{mM}$ sodium EDTA and $50 \mathrm{mM}$ sodium acetate, $\mathrm{pH} 6.5$. Ten microliters $(0.05$ to $0.10 \mathrm{mg}$ protein) of hepatocyte membrane extract in the homogenization buffer was added to a total volume of $50 \mu$ l. The reaction was initiated by addition of $4 \mu \mathrm{M}\left[{ }^{3} \mathrm{H}\right] \mathrm{SAM}$ and incubated for $30 \mathrm{~min}$ at $37^{\circ}$. The assay was terminated by addition of $3 \mathrm{ml}$ chloroform-methanol- $\mathrm{HCl}$ (2/1/0.02, by vol.) and $2 \mathrm{ml}$ of $0.1 \mathrm{M} \mathrm{KCl}$ in $50 \%$ methanol. The tubes were shaken for $15 \mathrm{~min}$ and centrifuged for $10 \mathrm{~min}$ at $2000 \mathrm{~g}$. Following aspiration of the aqueous phase, the chloroform layer was rewashed with $2 \mathrm{ml}$ of $0.1 \mathrm{M} \mathrm{KCl}$ in $50 \%$ methanol; 
Table 1. Methyltransferase activity in inbred and $\mathrm{H}-2$ congenic strains of mice

\begin{tabular}{|c|c|c|c|c|c|c|}
\hline Strain* & $\mathbf{N}$ & $\begin{array}{c}\text { MT I activity } \\
\text { (fmoles/ } / \text { g protein) }\end{array}$ & $\mathbf{N}$ & $\begin{array}{c}\text { MT II activity } \\
\text { (fmoles } / \mu \text { g protein) }\end{array}$ & Background & $H-2$ type \\
\hline \multicolumn{7}{|l|}{ Inbred } \\
\hline $\mathrm{A} / \mathrm{J}$ & 6 & $10.03 \pm 0.50 \dagger$ & 4 & $409.4 \pm 9.14 \dagger$ & A & a \\
\hline C57BL/6J & 6 & $19.44 \pm 0.72$ & 4 & $392.8 \pm 3.81$ & C57BL(B) & $\mathrm{b}$ \\
\hline C57BL/10Sn & 2 & $20.20 \pm 0.40$ & & NT $\ddagger$ & B & b \\
\hline $\mathrm{DBA} / 2 \mathrm{~J}$ & 5 & $15.57 \pm 0.12$ & 2 & $407.2 \pm 25.0$ & DBA & $\mathrm{d}$ \\
\hline $\mathrm{BALB} / \mathrm{cJ}$ & 6 & $15.24 \pm 0.64$ & 2 & $386.4 \pm 36.2$ & $\mathrm{BALB} / \mathrm{c}$ & d \\
\hline AKR & 5 & $24.98 \pm 0.12$ & 2 & $509.0 \pm 28.6$ & AKR & k \\
\hline $\mathrm{C} 3 \mathrm{H}$ & 5 & $25.54 \pm 1.42$ & & NT & $\mathrm{C} 3 \mathrm{H}$ & $\mathrm{k}$ \\
\hline $\mathrm{C} 57 \mathrm{BR} / \mathrm{cdJ}$ & 4 & $25.52 \pm 0.28$ & & NT & C57BR & k \\
\hline A/WYSn & 6 & $20.96 \pm 0.18$ & 2 & $417.0 \pm 46.9$ & $\mathrm{~A} / \mathrm{WY}$ & a \\
\hline \multicolumn{7}{|l|}{ Congenic } \\
\hline A.SW & 3 & $15.07 \pm 0.61$ & & NT & A & s \\
\hline A.BYSn & 4 & $20.58 \pm 1.40$ & 2 & $421.0 \pm 62.5$ & A/WY & b \\
\hline B10.A & 4 & $9.50 \pm 1.03$ & $\overline{2}$ & $464.3 \pm 50.0$ & B & $\mathrm{a}$ \\
\hline B10.S & 5 & $14.99 \pm 0.41$ & & NT & $\mathrm{B}$ & s \\
\hline B10.BR & 3 & $25.31 \pm 0.64$ & & NT & B & $\mathrm{k}$ \\
\hline B10.D2 & 3 & $15.84 \pm 0.18$ & & NT & B & d \\
\hline
\end{tabular}

* Letters and numerals before a slash or period indicate the strain; numerals and letters after a slash indicate sublines and/or holders while numerals and letters after a period indicate the source of the introduced gene.

$\dagger$ Mean \pm S.E.

$\ddagger$ Not tested.

$1 \mathrm{ml}$ of the resulting chloroform phase was dried in a scintillation vial at $60^{\circ}$ and counted with Instagel scintillation fluid. All the samples were assayed in duplicate and averaged.

MT II activity was assayed with $50 \mathrm{mM}$ sodium borate buffer, $\mathrm{pH} 10,1 \mathrm{mM}\left[{ }^{3} \mathrm{H}\right] \mathrm{SAM}$ and $100 \mu \mathrm{g}$ PMME by the procedure described above. The two products, phosphatidyldimethylethanolamine (PDME) and phosphatidylcholine (PC), were counted.

To visualize reaction products, the chloroform phase was dried under nitrogen gas to prevent oxidation and the residue was dissolved in $50 \mu$ chloroform-methanol $(2 / 1, v / v)$. The samples were applied to silica gel $\mathrm{G}$ chromatograph plates and developed in chloroform-methanol- $\mathrm{H}_{2} \mathrm{O}(75 / 24 / 4$, by vol.). Commercially available markers of PE, PMME, PDME, and PC were cochromatographed with the samples, and the spots were visualized by spraying with $0.06 \%$ Rhodamine $6 \mathrm{G}$ solution.

$S$-Adenosyl-L- $\left[\right.$ methyl $\left.-{ }^{3} \mathrm{H}\right]$ methionine $\quad(5-15 \mathrm{Ci} /$ mmole) was purchased from ICN, Irvine, CA. $\left[{ }^{3} \mathrm{H}\right]-$ SAM $(100-500 \mathrm{mCi} / \mathrm{mmole})$ was obtained from Amersham, Arlington Heights, IL. S-Adenosyl-Lhomocysteine, PE, PMME, PDME, and PC were obtained from the Sigma Chemical Co., St. Louis, MO. All other reagents were from Mallinckrodt, Paris, KY.

\section{RESULTS}

Methyltransferase I activity was determined as a function of the incorporation of $4 \mu \mathrm{M}\left[{ }^{3} \mathrm{H}\right] \mathrm{SAM}$ into endogenous PE forming PMME. The radioactivity was quantified directly by counting the chloroform layer of the extraction, and the rate of reaction was found to be linear with time up to $40 \mathrm{~min}$. PMME was visualized by thin-layer chromatography and, after scraping and counting of the spot, was found to contain approximately $75 \%$ of total radiolabel applied to the silica plate, i.e. $25 \%$ of the radioactivity in the chloroform extract was not recovered by the scraping procedure (or consisted of other compounds).

Results of MT I assays on hepatocyte membranes from various inbred mouse strains are shown in Table 1 . Of the nine inbred strains studied, $\mathrm{A} / \mathrm{J}$ showed the lowest MT I activity, while AKR and $\mathrm{C} 3 \mathrm{H}$ had the highest. The remainder (C57BL/6J, DBA/2J, A/WYSn, and A.SW) were intermediate in their MT I activity. Lineweaver-Burk plots of various concentrations of $\left[{ }^{3} \mathrm{H}\right] \mathrm{SAM}$ as an indirect measurement of substrate concentration provided preliminary data that the differences in MT I activity were due to differences in apparent $V_{\max }$. The $K_{m}$ value appeared to be the same for all the strains (data not shown).

We then measured MT I activity in hepatocyte membranes from $H-2$ congenic mouse strains. These results are also presented in Table 1. Four of the strains had varying haplotypes $\left(H-2^{\mathrm{a}}, H \cdot 2^{\mathrm{d}}, H \cdot 2^{\mathrm{s}}\right.$, and $H-2^{\mathrm{k}}$ ) on a C57BL genetic background, while one, A-BYSn, carried the $H-2^{\mathrm{b}}$ haplotype on the A/WY background. In all five strains, the level of enzyme activity corresponded to that of the donor $\mathrm{H}$ 2 haplotype. One inbred strain, A/WYSn, however, was found to have a higher level of MT I activity than the other $H-2^{\text {a }}$ haplotypes.

To localize the effects of $H-2$ on MT I activity observed in $\mathbf{H}-2$ congenics to a particular region of the major histocompatibility complex, we assayed several recombinant haplotypes for this enzyme. The results are shown in Table 2 . The increase in activity in the B10.A(3R), (4R), and (5R) hepatocyte membranes compared with those from the $B 10$. A animals suggests that the $\mathrm{K}$ end of the $H \cdot 2$ complex is 
Table 2. Methyltransferase I activity in recombinant $H-2$ haplotypes

\begin{tabular}{lccccc}
\hline Strain & N & $\begin{array}{c}\text { MT I activity } \\
\text { (fmoles/ } \mu \text { g protein) }\end{array}$ & Inbred partner & Donor strain & $\begin{array}{c}\text { Haplotype of origin } \\
\text { K A E S D }\end{array}$ \\
\hline A/J* & 6 & $10.03 \pm 0.50 \dagger$ & & & k k k d d \\
C57BL/6,10Sn & 8 & $19.63 \pm 0.55$ & & & b b b b \\
B10.A & 4 & $9.50 \pm 1.03$ & C57BL10/Sn & A/WY & k k d d \\
B10.A(5R) & 4 & $20.89 \pm 0.51$ & C57BL10/Sn & A/WY & b k d d \\
B10.A(4R) & 3 & $25.78 \pm 0.25$ & C57BL10/Sn & A/WY & k k b b \\
B10.A(3R) & 3 & $18.64 \pm 0.78$ & C57BL10/Sn & A/WY & b k d d \\
B10.D2 & 3 & $15.84 \pm 0.18$ & C57BL10/Sn & DBA/2J & d d d d d \\
B10.D2-H-2 & 1 & $5.80 \pm 0.11$ & C57BL10/Sn & B10.D2 & d d d - \\
A.TL & 3 & $15.95 \pm 0.52$ & A/SnSf & A.SW & s k k d \\
A.TH & 3 & $15.34 \pm 0.27$ & A/SnSf & B10.S & s s s d d \\
\hline
\end{tabular}

* MT I activity in the A/J strain appeared to be mediated by the effects of other non- $H-2$ genes of the A genetic background. This is suggested by the observation that, although $\mathrm{A} / \mathrm{J}$ is a natural $\mathrm{k} / \mathrm{d}$ recombinant haplotype, its $\mathrm{MT}$ $\mathrm{I}$ activity was nevertheless low, atypical of other $\mathrm{K}$ haplotypes.

$\dagger$ Mean \pm S.E.

involved in the regulation of MT I activity. This is supported by the change in activity observed in the A.TL mice compared to the B10.BR strain. Crossovers at the $\mathrm{D}$ end of the $\mathrm{H}-2$ complex do not appear to affect MT I activity, as illustrated by the A.TH recombinant haplotype and the B10.D2-H-2 mutant.

Methyltransferase II activity was assayed by measuring the incorporation of tritium from $\left[{ }^{3} \mathrm{H}\right] \mathrm{SAM}$ into exogenous PMME in the chloroform phase. The reaction products, PDME and $\mathrm{PC}$, were visualized by thin-layer chromatography and found to have incorporated approximately 25 and $55 \%$ of total radiolabel, respectively, after $30 \mathrm{~min}$ of incubation. The reaction was determined to be linear with time up to $40 \mathrm{~min}$. No significant differences were found among the inbred strains studied (Table 1). The Lineweaver-Burk plots for the $\mathrm{A} / \mathrm{J}$ and $\mathrm{C} 57 \mathrm{BL} / 6 \mathrm{~J}$ strains did not show significant differences in the apparent $V_{\max }$ or $K_{m}$ (data now shown).

\section{DISCUSSION}

Methylation of membrane phospholipids is mediated by two specific methyltransferases [7]. The first enzyme (MT I) methylates phosphatidylethanolamine to phosphatidylmonomethylethanolamine, requires magnesium, has a high affinity for $S$-adenosyl-L-methionine, and is believed to be located in the inner leaflet of the plasma membrane. The second enzyme (MT II) subsequently transfers two methyl groups to PMME to form phosphatidylcholine, has a low affinity for $S$-adenosyl-Lmethionine, does not require magnesium, and is believed to be located in the outer leaflet. The asymmetric distribution of these enzymes and their substrates within the membrane facilitates the translocation of phospholipids across the matrix and results in changes in membrane fluidity [8]. These alterations in membrane fluidity have been postulated to affect the coupling of $\beta$-adrenergic receptors to adenylate cyclase [3]. Phosphatidylcholine synthesis by transmembrane methylation appears to be under the control of several other hormones, including glucagon [9], angiotensin, and vasopressin
[10], which modulate the activation of adenylate cyclase. Not all receptor-mediated processes, however, interact with this series of methylation reactions. Stimulation of platelets, for example, with thrombin, prostaglandin, or epinephrine, was without effect on transmethylation in these cells [3]. Phospholipid methylation was also found to be nonessential in amylase secretion and activation of adenylate cyclase by $\beta$-adrenergic agonists in the guinea pig and rat parotid gland [11]. Since $\beta$-adrenergic stimulation of adenylate cyclase has been shown to depend on transmembrane methylation in other systems, species- and tissue-specific differences may be involved in the extent of interaction between phospholipid methylation and receptor-mediated response.

Glucagon-stimulated adenylate cyclase activity has been found to be under the influence of the mouse major histocompatibility complex, $H-2$ [2]. Since the mode of action of glucagon in liver is analogous to that of the $\beta$-adrenergic catecholamines, we investigated the possible effects of the MHC on phospholipid methyltransferase activity in hepatocyte plasma membranes from inbred and $\mathrm{H}-2$ congenic strains of mice.

While phospholipid methyltransferase activity is also found in microsomal $(27.4 \pm 1.92 \mathrm{pmoles} / \mathrm{mg}$ protein $/ 30 \mathrm{~min})$ and mitochondrial $(9.82 \pm 0.28$ pmoles $/ \mathrm{mg}$ protein $/ 30 \mathrm{~min}$ ) fractions of rat and bovine brain, the highest specific activity is localized to the plasma membrane $(28.0 \pm 0.73 \mathrm{pmoles} / \mathrm{mg}$ protein/30 $\mathrm{min}$ ) [12]. We utilized a 10 -fold purified plasma membrane fraction of mouse liver to investigate methyltransferase activity in inbred and $\mathrm{H}-2$ congenic strains. Variations in relative recoveries of components may contribute to, but do not fully explain, the variations in enzymatic activity that we found, since we found variations between strains that were as large as those between subcellular fractions, i.e. the plasma membrane fraction would have to consist entirely of one contaminant in one strain and entirely of another component in another strain.

By measuring the incorporation of radioactivity from $\left[{ }^{3} \mathrm{H}\right] \mathrm{SAM}$ into endogenous $\mathrm{PE}$, we found significant variation among inbred strains of mice in 
MT I activity (Table 1). Preliminary data suggest that this variation is due to differences in apparent $V_{\max }$ while the $K_{m}$ values remained the same. Variations in levels of endogenous substrate (PE) in the membranes are unlikely to contribute to the results since PE represents $20 \%$ of phospholipid, or $11 \%$ of total lipid, of mouse liver membranes [13], providing a large excess of endogenous substrate. Alternatively, strain variation in levels of methyltransferase inhibitors could contribute to the variations in enzymatic activity found.

MT I activity was subsequently measured in hepatocyte membranes from $H-2$ congenic strains. In five of the six strains tested, the level of methyltransferase activity corresponded to that of the donor $H$-2 haplotype. The A/WYSn strain, however, showed a higher level of enzyme activity than did the other strains with the $H-2^{\mathrm{a}}$ haplotype. A possible explanation for this discrepancy might be attributed to the effects of genetic background on MT I. Studies with congenic lines focus on the effects of a single locus or small chromosomal segment (3-10 centiMorgans, in the case of $H-2$, [14]) on a particular phenotype, while the genetic background is kept constant. Under such circumstances, $H-2$ exerts significant effects on MT I activity. However, the $\mathrm{A} / \mathrm{J}$ and $\mathrm{A} / \mathrm{WYSn}$ strains, both originally of the $\mathrm{A}$ background, have been separated for over 100 generations. Even though both are $H-2^{\text {a }}$, the variation in the genetic backgrounds of these two strains could account for the difference in MT I activity. In their studies of the $H$ 2 influence on liver cyclic AMP levels, Lafuse and co-workers [1] found a similar trend in strains of background $\mathrm{A}$. These investigators concluded that the effect contributed by other (non- $H-2$ ) loci on this background overrode the effects of the MHC on glucagon responses. Interestingly, as seen in Table 1 , the basal MT I activities in the inbred and congenic strains all vary by increments of about five activity units. This may or may not be significant for the biochemical mechanisms of action of MT I.

Since Edidin and co-workers [1,2] investigated the effects of $\mathrm{H}-2$ on glucagon-stimulated cyclic AMP levels and an interaction exists between glucagon binding and membrane phospholipid $\mathrm{N}$-methylation, we compared our results with theirs, finding a striking pattern. This is illustrated in Fig. 1. $H-2^{a}$, the haplotype with the highest level of liver cyclic AMP and the lowest $K_{m}$ for glucagon, had the lowest MT I activity, while the haplotype with the highest MT I activity $\left(H-2^{\mathrm{k}}\right)$ had the lowest cyclic AMP levels and the highest $K_{m}$ for glucagon $(r=0.88)$. The others $\left(H-2^{\mathrm{b}}, H-2^{\mathrm{d}}\right.$ and $\left.H-2^{\mathrm{s}}\right)$ were intermediate for all three variables. Furthermore, from their results of $H \cdot 2$ recombinants, Lafuse et al. [1] concluded that at least two $H-2$ associated loci affect intracellular cyclic AMP levels, one of these mapping to the $K$ end of the MHC. Our investigations using recombinant haplotypes indicated that this region is also involved in the control of MT I activity (Table 2). The exception lies with the $\mathrm{A} / \mathrm{J}$ strain which is a natural $\mathrm{k} / \mathrm{d}$ recombinant haplotype. The $\mathrm{K}$ end is $\mathrm{k}$, and yet A/J showed low basal MT I (unlike $H-2^{\mathrm{k}}$ ). This discrepancy may be attributed to the effects of other, non- $H-2$, genes previously observed in the A genetic background. The inbred partner of the series of crosses resulting in the A.TL and A.TH strains is $\mathrm{A} / \mathrm{SnSF}$. We were unable to obtain this strain. However, A/Sn has been separated from A/WYSn for only $15-20$ generations [15]. Since C57BL/10J and

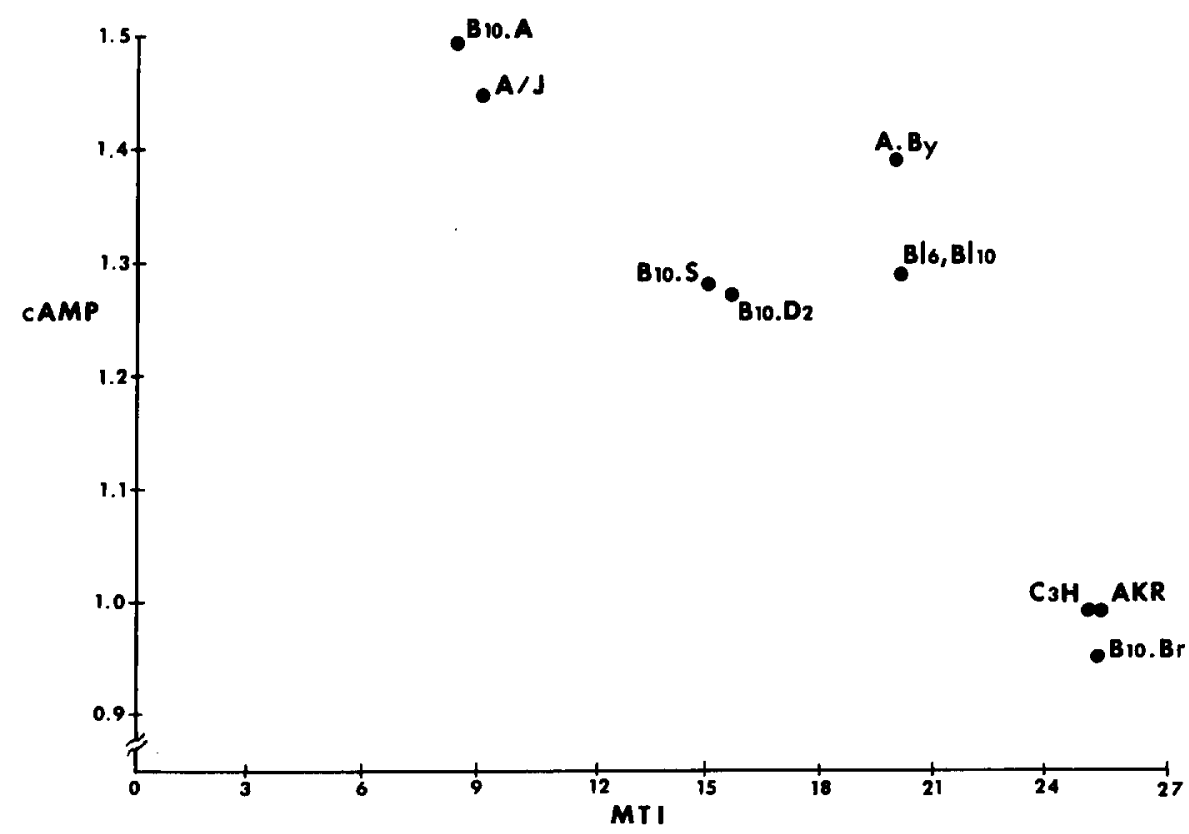

Fig. 1. Plot of MT I activity versus intracellular cyclic AMP levels in inbred and $H-2$ congenic mice. Methyltransferase I activities are those shown in Table 1. Cyclic AMP values, expressed as pmoles $\mathrm{cAMP} / \mathrm{mg}$ wet liver weight, are as given by Lafuse et al. [1]. 
C57BL/10Sn have been separated for over 30 generations [15] with no change in Mt I activity between the two, we assume that $\mathrm{A} / \mathrm{Sn}$ and $\mathrm{A} / \mathrm{WYSn}$ have not diverged sufficiently to affect the activity of this enzyme.

While Hirata et al. [8] and Castaño et al. [9] reported that phospholipid methylation increases following $\beta$-adrenergic and glucagon stimulation, respectively, the negative correlation of our results with those of Lafuse and coworkers does not contradict their findings. We determined basal levels of MT I activity without hormonal stimulation. Lower basal levels of membrane methyltransferase I might show a greater response to hormone-induced changes to achieve the same, or higher, end states. Preliminary results indicate that MT I activity is stimulated to the same levels by isoproterenol in the $\mathrm{A} / \mathrm{J}$ and $\mathrm{C57BL} / 6 \mathrm{~J}$ strains [16]. Alternatively, continued in vivo stimulation could have led to greater "down regulation" in the more sensitive strain.

Our investigations do not eliminate the possibility that $H-2$ may exert an effect on the endogenous levels of phosphatidylethanolamine in the cell membranes and, thus, contribute indirectly to the regulation of MT I, although it is unlikely given the high concentration of $\mathrm{PE}$ in mouse liver plasma membranes. Furthermore, the MHC may also be involved in the production of possible stimulatory or inhibitory modulators of MT I in certain strains. Although the biochemical role of the $\mathrm{MHC}$ in the regulation of $\mathrm{MT}$ I is not clear, striking correlations emerge between biological signals associated with $H-2$ or $H L A$ and those apparently mediated by phospholipid methylation. Erickson et al. (manuscript in preparation) have found an $H L A$ association with the degree of lectin stimulation of lymphocytes. Increased phospholipid methyltransferase activity has been reported in analogous systems following stimulation with concanavalin A $[17,18]$. Similarly, $H-2$ antigens have been shown to influence killer $\mathrm{T}$ cell specificity and function [19], while the methylation of membrane phospholipids markedly increases during cytotoxicity by these cells [20]. Correlations such as these, as well as that presented in this paper, suggest the possibility that differences in methyltransferase activity are involved in mediating those MHC associations.

Acknowledgement-This work was supported by grants (GM 27028 and DE 06108) from the National Institutes of Health.

\section{REFERENCES}

1. W. Lafuse, D. Meruelo and M. Edidin, Immunogenetics 9, 57 (1979).

2. W. Lafuse and M. Edidin, Biochemistry 19, 49 (1980).

3. F. Hirata and J. Axelrod, Science 209, 1083 (1980).

4. F. Hirata, O. H. Viveros, E. J. Diliberto, Jr. and J. Axelrod, Proc. natn. Acad. Sci. U.S.A. 75, 1718 (1978).

5. S. Yamashita, A. Oshima, J. Nikawa and K. Hosaka, Eur. J. Biochem. 128, 589 (1982).

6. G. A. Scarborough and J. F. Nye, J. biol. Chem. 242, 238 (1967).

7. F. Hirata and J. Axelrod, Proc. natn. Acad. Sci. U.S.A. 75, 2348 (1978).

8. F. Hirata, W. J. Strittmatter and J. Axelrod, Proc. natn. Acad. Sci. U.S.A. 76, 368 (1979).

9. J. Castaño, S. Alemany, A. Nieto and J. Mato, J. biol. Chem. 255, 9041 (1980).

10. S. Alemany, 1. Varela and M. M. Mato, Fedn. Eur. Biochem. Soc. Lett. 135, 111 (1981).

11. U. Padel, C. Unger and H. Soling, Biochem. J. 208, 205 (1982).

12. F. T. Crews, F. Hirata and J. Axelrod, J. Neurochem. 34, 1491 (1980).

13. R. van Hoeven and P. Emmelot, J. membr. Biol. 9, 105 (1972).

14. D. Klein, S. Tewarson, F. Figueroa and J. Klein, Immunogenetics 16, 319 (1982).

15. P. L. Altman and D. Dittmer-Katz (eds.), Inbred and Genetically Defined Strains of Laboratory Animals, Part I: Mouse and Rat, p. 18. Fed. Amer. Soc. Exp. Biol., Bethesda, MD (1979).

16. J. Markovac and R. P. Erickson, Genet. Res., in press.

17. F. Hirata, J. Axelrod and F. T. Crews, Proc. natn. Acad. Sci. U.S.A. 76, 4813 (1979).

18. F. Hirata, S. Toyoshima, J. Axelrod and M. J. Waxdal, Proc. natn. Acad. Sci. U.S.A. 77, 862 (1980).

19. P. J. Fink and M. J. Bevan, Proc. natn. Acad. Sci. U.S.A. 78, 6401 (1981).

20. T. Hoffman, F. Hirata, P. Bougnoux, B. A. Fraser, R. H. Goldfarb, R. B. Herberman and J. Axelrod, Proc. natn. Acad. Sci. U.S.A. 78, 3839 (1981). 